\title{
CLEO'OL
}

\section{Direct microstructuring of fiber cores with femtosecond laser pulses}

\author{
John D. Mills, Peter G. Kazansky, Erica Bricchi and Chris W. J. Hillman \\ Optoelectronics Research Centre \\ Jeremy J. Baumberg \\ Department of Physics and Astronomy

\begin{abstract}
Periodic structures are directly written into the core of single-mode optical fibers with a femtosecond laser enabling control over the exiting light. The diffracting beams retain their original mode profile offering new device possibilities.
\end{abstract}

Recently, the use of a focused femtosecond laser source to directly write structures deep within glass has attracted much attention due to its simplicity compared to lithographic methods, and its ability to write in three-dimensions $[1,2]$. We have developed a novel technique, which enables us to write such structures within the bulk of an optical fiber through its cleaved face, enabling control over light subsequently exiting the fiber. Figure 1 displays the experimental set-up where an amplified Ti:Sapphire $150 \mathrm{fs}$ pulsed laser with a repetition rate of $250 \mathrm{kHZ}$ is used to irradiate the samples at $850 \mathrm{~nm}$, via a $50 \mathrm{x}$ objective. The typical pulse energy utilized is $0.5 \mu \mathrm{J}$. Initial positioning of the focal spot of the laser relative to the core of the fiber is achieved by maximizing the guiding of the pulsed light through the fiber at an intensity below damage threshold [3]. Observation of the processing subsequently written into the core and cladding regions of the fiber can be monitored in situ via a coaxial CCD system. Altematively, the CCD camera can been used to monitor the effects of material damage on the mode during irradiation, by coupling a laser to the opposite end of the fiber, in place of the photo-diode.

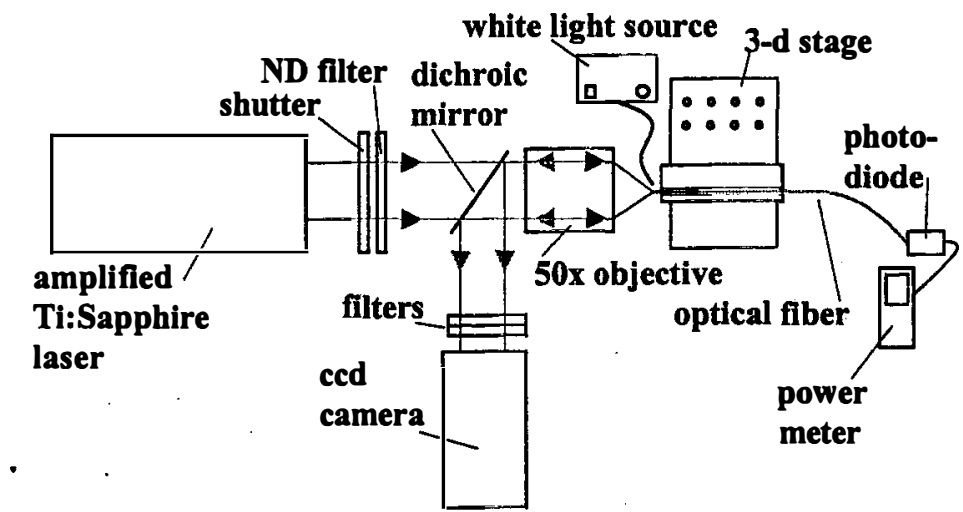

Fig. 1. Schematic of the optical fiber direct-write set-up

Figure 2 shows a microscope image of an embedded diffraction grating having a $5 \mu \mathrm{m}$ pitch and buried $25 \mu \mathrm{m}$ below a fiber's cleaved face. The grating, which spans the cross-section of the $125 \mu \mathrm{m}$ diameter fiber, has been illuminated for the purpose of identification by allowing white light to be guided from the fiber's opposite end. One and two-dimensional embedded gratings as close as $5 \mu \mathrm{m}$ to the cleaved surface with a pitch of just $1.4 \mu \mathrm{m}$ have been successfully written into small-core fibers, to induce diffraction. Fiber that is able to support several modes has also been successfully tested, confirming mode profile preservation within each of its diffracting orders. 


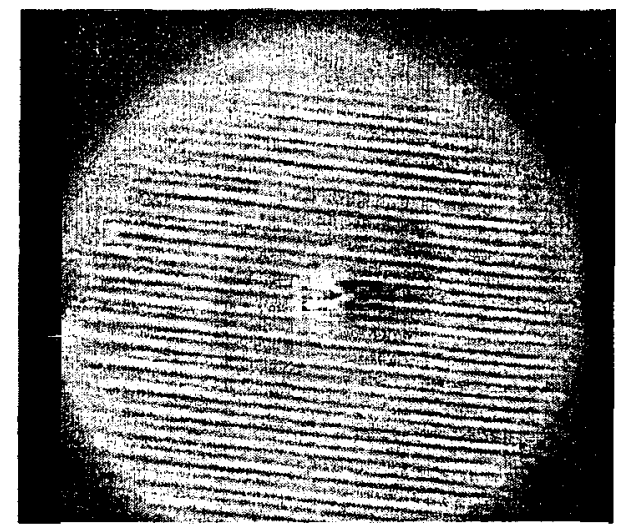

Fig. 2. A $5 \mu \mathrm{m}$ period embedded diffraction grating directly written $25 \mu \mathrm{m}$ below a fiber's cleaved face

Figure 3 displays a far-field pattern created by a single--mode fiber guiding HeNe light at $632.8 \mathrm{~nm}$, and showing the zeroth and first diffracting orders. The pattern in this case has been generated with a two-dimensional grating, consisting of a $20 \times 20$ array of dots, which was produced by irradiating the $6 \mu \mathrm{m}$ diameter core region, $5 \mu \mathrm{m}$ below its surface. The writing laser's electronic shutter was open for $4 \mathrm{~ms}$ at each point, equating to approximately 1000 pulses. The period of the array was $1.4 \mu \mathrm{m}$ and was therefore confined to the central core region of the fiber.

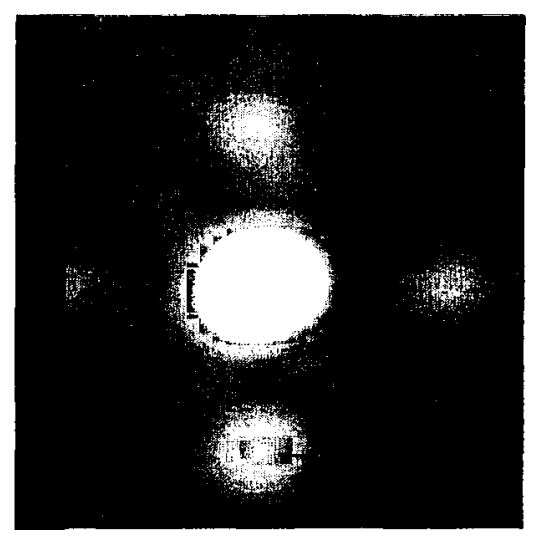

Fig. 3. CCD recorded far-field pattem of HeNe light exiting a single-mode fiber. The diffraction pattem was created with an array of $20 \times 20$ dots, with a regular spacing of $1.4 \mu \mathrm{m}$, centred upon the fiber core's axis and embedded $5 \mu \mathrm{m}$ below the fiber's cleaved face

The controllability of the power and direction of diffracting orders offers tantalizing opportunities for new methods of optical routing. Additionally, we are investigating the possibility of designing evanescent field related devices by characterizing the local field distribution of light exiting fibers, with Scanning Near-field Optical Microscopy (SNOM) [4].

[1] K.M. Davis, K.Miura, N. Sugimoto, K. Hirao, “Writing wave-guides in glass with a femtosecond laser,"Opt. Lett. 21, 1729-1731 (1996) [2] A.M. Strelsov, N.F. Borrelli, "Fabrication and analysis of a directional coupler written in glass by nanojoule femtosecond laser pulses," Opt. Lett. 26, $42-43$ (2001)

[3] M. Lenzner, J. Kruger, S. Sartania, Z. Cheng, Ch. Spielmann, G. Mourou, W. Kautek, F. Krausz, "Femtosecond optical breakdown in dielectrics," Phys. Rev. Lett. 80, 4076-4079 (1998)

[4] J.D. Mills, C.W.J. Hillman, W.S. Brocklesby, B.H. Blott, "Evanescent field imaging of an optical fiber Bragg grating," Appl. Phys. Lett. 75, 4058-4060 (1999) 